\title{
Non-Vitamin K Oral Anticoagulants in Stroke Patients: Practical Issues
}

\author{
Konark Malhotra, ${ }^{a}$ Monica Khunger, ${ }^{\mathrm{b}}$ David S Liebeskind ${ }^{\mathrm{a}}$ \\ aUCLA, Los Angeles, United States \\ ${ }^{b}$ Cleveland Clinic, Cleveland, United States
}

Dear Sir:

We would like to congratulate the authors for their welldrafted article published in the May 2016 issue entitled "NonVitamin K Oral Anticoagulants in Stroke Patients: Practical Issues.'. We read the article with interest and would like to humbly highlight few of our observations and comments from our experience.

\section{Significance of imaging}

The concept of "1-3-6-12 day rule" was recently mentioned as a consensus approach for recommendations for anticoagulation in patients with cerebral ischemic event, either transient ischemic attack or stroke. ${ }^{2}$ As mentioned by the authors, the initiation of anticoagulation in these patients depends on various factors. However, the decision to initiate or restart the anticoagulation primarily pivots around the size of infarction delineated by neuroimaging. Recent advent of novel imaging techniques has been critical in the success in stroke community. ${ }^{3}$ Risk of cerebral hemorrhagic transformation is majorly related to the size and age of the cerebral infarct. In our clinical practice, we tend to prefer following simplified, yet logical algorithm to approach patients with recent cerebral ischemic event who have a strong indication for anticoagulation (Table 1): A) No infarction or clinically diagnosed transient ischemic attack: anticoagulation could be started immediately. B) Infarct size $\leq 1.5 \mathrm{~cm}$ diameter $\rightarrow$ anticoagulation could be started in 2 days, C) Infarct size between $1.6 \mathrm{~cm}-3 \mathrm{~cm} \rightarrow$ anticoagulation to be initiated in 4-5 days, D) Infarct size $\geq 3 \mathrm{~cm}$ : anticoagulation could be started at day 7 .
Any patients with hemorrhagic conversion or large parenchymal bleeds should be reimaged to confirm the stability of bleed. For cases with mild hemorrhagic infarction ( $\mathrm{HI}-1 / 2)$, anticoagulation could be started in 7-10 days, while more severe cerebral hemorrhage including parenchymal hematomas, subdural or subarachnoid hemorrhage require a minimum waiting time of at least 2-4 weeks depending on urgency of anticoagulation in individual cases.

\section{Significance of stroke severity}

Authors tend to describe the terminology of mild stroke as patients who present with National Institute of Health Stroke Scale (NIHSS) $\leq 8$ in their article. However, we beg to defer as in stroke community, mild or minor stroke generally refer to patients who present with stroke symptoms with severity that accounts for $\mathrm{NIHSS} \leq 5 .{ }^{4}$ Though referred as mild or minor stroke in terminology, around $33 \%$ of patients in this subclass suffer with poor outcomes. We suggest usage of terminology that could be easily perceived and readily implemented by the stroke community.

\section{Significance of emergency medications}

Various procoagulants have been used in emergent situations to reverse vitamin $\mathrm{K}$ antagonists related intracranial hemorrhage (VKA-ICH) including prothrombin complex concentrate, activated prothrombin complex concentrate or Factor Eight Inhibitor Bypassing Activity (FEIBA), fresh frozen plasma and recombinant Factor VIla. VKA such as warfarin acts on both intrinsic and extrinsic clotting factors. ${ }^{5}$ Thus, the physicians tend to favor use of procoagulants for Vitamin $\mathrm{K}$ antagonist related intracranial 
Table 1. Algorithm for initiation of anticoagulation (if indicated) after acute ischemic or hemorrhagic stroke

Neuroimaging characteristics in stroke patients Initiation of anticoagulation*

A) No infarction or clinically diagnosed TIA Anticoagulation could be started immediately.

B) Infarct size $\leq 1.5 \mathrm{~cm}$ diameter Anticoagulation could be started in 2 days.

C) Infarct size between $1.6 \mathrm{~cm}-3 \mathrm{~cm}$ Anticoagulation could be initiated in 4-5 days.

D) Infarct size $\geq 3 \mathrm{~cm}$ Anticoagulation could be started at day 7

E) Hemorrhagic transformation (Hi-1/2) Anticoagulation could be stated in 7-10 days, require prior repeat neuroimaging*

F) Parenchymal hematomas/Subdural or Subarachnoid hemorrhage Hold off anticoagulation for a minimum of 2-4 weeks, require prior repeat neuroimaging ${ }^{\dagger}$

*Initiation of anticoagulation usually depends on the urgency of anticoagulation based on each individual case; ${ }^{\dagger}$ All large ischemic stroke cases and especially hemorrhage cases require neuroimaging prior to anticoagulation initiation.

hemorrhage to replete the concentration of activated or non-activated forms of such clotting factors. However, one of their major side effects of these procoagulants include predisposition to thromboembolic events that has been a major concern in these situations. The combination of these procoagulants should be avoided in routine clinical practice. We recommend use of either procoagulants to avoid development of hypercoagulable state in these critical patients, with a preference for prothrombin complex concentrate from recent data as mentioned below. Recent advent of antidotes for non-vitamin $\mathrm{K}$ antagonist oral anticoagulants has opened up new horizon, though data for their utilization in reversal of Vitamin $\mathrm{K}$ antagonist related intracranial hemorrhage remain scarce.

\section{Significance of new study results}

As mentioned by the authors, data on the efficacy of various procoagulants for the treatment of anticoagulant related ICH remains scarce. However, recently published international normalized ratio normalization in patients with coumarin-related intracranial hemorrhages (INCH) study has demonstrated the superiority of four-factor prothrombin complex concentrate over fresh frozen plasm in respect to international normalized ratio normalization and hematoma expansion. ${ }^{6}$ Though there was no benefit in relation to clinical outcome, the study clearly guides clinicians for rapid normalization of international normalized ratio and control of hematoma expansion in emergent situations.

\section{Significance of drug pharmacokinetics}

Authors in their article have briefly mentioned the varying degrees of elimination for various non-vitamin $\mathrm{K}$ antagonist oral anticoagulants with 33\% of Rivaroxaban accounting for renal excretion. We would further like to highlight over the pharmacokinetic details of this drug as $\sim 1 / 3$ part of the drug is excreted as active or unchanged form in the urine, while remaining $\sim 2 / 3$ parts of the drug is eliminated as inactive metabolites in the form of both urine and feces.?
Significance of anticoagulation in elderly with falls

Use of anticoagulation in elderly patients who are prone to falls poses a therapeutic challenge in decision-making for physicians and as mentioned by the authors, and this issue needs to be discerned for each individual patient. Few previous studies have estimated that an average person needs to have approximately 300 falls per year to be disqualified from anticoagulation therapy, especially VKA. ${ }^{8}$ Though this could be applied as a general rule to support physicians to arrive at therapeutic equipoise, however many of these cases are challenging and require supreme caution.

\section{References}

1. Diener $\mathrm{HC}$, Kleinschnitz $\mathrm{C}$. Non-vitamin $\mathrm{K}$ oral anticoagulants in stroke patients: practical issues. J Stroke 2016;18:138-145.

2. Heidbuchel $H$, Verhamme $P$, Alings $M$, Antz $M$, Diener $H C$, Hacke W, et al. Updated European Heart Rhythm Association Practical Guide on the use of non-vitamin K antagonist anticoagulants in patients with non-valvular atrial fibrillation. Europace 2015;17:1467-1507.

3. Malhotra K, Liebeskind DS. Imaging in endovascular stroke trials. J Neuroimaging 2015;25:517-527.

4. Vagal AS, Sucharew $H$, Prabhakaran $S$, Khatri $P$, Jovin T, Michel $P$, et al. Final infarct volume discriminates outcome in mild strokes. Neuroradiol J 2015;28:404-408.

5. Lazzaro MA, Malhotra K, Mohammad YM. The role of antithrombotics in secondary stroke prevention. Semin Neurol 2010; 30:492-500.

6. Steiner T, Poli S, Griebe M, Hüsing J, Hajda J, Freiberger A, et al. Fresh frozen plasma versus prothrombin complex concentrate in patients with intracranial haemorrhage related to vitamin $\mathrm{K}$ antagonists (INCH): a randomised trial. Lancet Neurol 2016;15:566-573.

7. Gonsalves WI, Pruthi RK, Patnaik MM. The new oral anticoagulants in clinical practice. Mayo Clin Proc 2013;88:495-511. 
8. Man-Son-Hing M, Nichol G, Lau A, Laupacis A. Choosing antithrombotic therapy for elderly patients with atrial fibrillation who are at risk for falls. Arch Intern Med 1999;159:677-685.
Correspondence: Konark Malhotra

UCLA, 757 Westwood Plaza, Los Angeles, United States

Tel: +4127580737

Fax: +3043886445

Email: konark.malhotra@yahoo.com

Received: July 21, 2016

Revised: August 18, 2016

Accepted: August 21, 2016

The authors have no financial conflicts of interest. 